\title{
Secondary electron emission yield on poled silica based thick films
}

\author{
Braga, D.; Poumellec, B.; Cannas, V.; Blaise, G.; Ren, Yitao; Kristensen, Martin
}

Published in:

Journal of Applied Physics

Link to article, DOI:

$10.1063 / 1.1758315$

Publication date:

2004

Document Version

Publisher's PDF, also known as Version of record

Link back to DTU Orbit

Citation (APA):

Braga, D., Poumellec, B., Cannas, V., Blaise, G., Ren, Y., \& Kristensen, M. (2004). Secondary electron emission yield on poled silica based thick films. Journal of Applied Physics, 96(1), 885-894.

https://doi.org/10.1063/1.1758315

\section{General rights}

Copyright and moral rights for the publications made accessible in the public portal are retained by the authors and/or other copyright owners and it is a condition of accessing publications that users recognise and abide by the legal requirements associated with these rights.

- Users may download and print one copy of any publication from the public portal for the purpose of private study or research.

- You may not further distribute the material or use it for any profit-making activity or commercial gain

- You may freely distribute the URL identifying the publication in the public portal

If you believe that this document breaches copyright please contact us providing details, and we will remove access to the work immediately and investigate your claim 


\title{
Secondary electron emission yield on poled silica based thick films
}

\author{
D. Braga \\ Physique des Solides, UMR8502 CNRS-UPS, Université de Paris Sud, F-91405 Orsay Cedex, France \\ B. Poumellec and V. Cannas \\ Laboratoire de Physico-Chimie de l'Etat Solide, UMR8648 CNRS-UPS, Universite de Paris Sud, \\ F-91405 Orsay Cedex, France \\ G. Blaise \\ Physique des Solides, UMR8502 CNRS-UPS, Université de Paris Sud, F-91405 Orsay Cedex, France
}

Y. Ren and M. Kristensen

Research Center COM, Technical University of Denmark, DK-2800 Kgs. Lyngby, Denmark

(Received 8 April 2003; accepted 10 April 2004)

\begin{abstract}
Studies on the distribution of the electric field produced by a thermal poling process in a layer of Ge-doped silica on silicon substrate, by using secondary electron emission yield (SEEY) measurements $(\delta)$ are presented. Comparing $\delta_{0}$ between poled and unpoled areas, the SEEY at the origin of electron injection, we pointed out an electric field $0.5 \mu \mathrm{m}$ below the surface for our poling conditions and directed in the same direction as the external field applied during the poling process. Then, the dependence of $\delta$ on the injected dose of electrons allows us to deduce that the poling process disturbs the glass structure strongly enough for leading to a weak conductivity. It is then easy to display the poled areas. We have also pointed out an effect of the electric properties of the glass on the measurements obtained with the Electron Probe for MicroAnalysis. (C) 2004 American Institute of Physics. [DOI: 10.1063/1.1758315]
\end{abstract}

\section{INTRODUCTION}

The poling is a process that allows a large internal electric field to be induced in an insulator by building a space charge to separate positive and negative charges. The usual experimental way is to deposit or apply electrodes on each side of a plate of insulator and to apply an electric field of the order of a few $\mathrm{V} / \mu \mathrm{m}$. Then, the insulator with electrodes is heated to about $300^{\circ} \mathrm{C}$ during $0.5 \mathrm{~h}-1 \mathrm{~h}$, cooled down, and the external electric field is removed. The insulator contains, then, a space charge formed by a layer of positive charges and another one negative. ${ }^{1}$ The charge distribution depends on the type and the mobility of the charge carrier at the poling temperature. So doing, in an insulator such as silica glass, the centrosymmetry is broken, and second-order nonlinear optical (NLO) properties are obtained. Especially, $\chi^{(2)}$ is now not zero. It is of the order of $0.1 \mathrm{pm} / \mathrm{V}$ in pure silica such as Infrasil that contains $\mathrm{Na}$ impurities. The $\chi^{(2)}$ is produced efficiently by the following formula $\chi^{(2)}=3 \chi^{(3)} E_{\mathrm{DC}}$, where $\chi^{(3)}$ is the third-order nonlinear coefficient (always nonzero) and $E_{\mathrm{DC}}$ is the electric field associated to the space charge. The glass exhibits also a second-order nonlinear refractive index by Pockel effect $\left(\sim \chi^{(3)} E_{\mathrm{DC}}\right.$, Ref. 7). With such properties, the glass is now able to double the frequencies and exhibits an index that can be modulated. It is thus possible to elaborate optical devices ${ }^{2,3}$ for optical information processing and telecommunication (e.g., switches, modulators, or routers).

The distribution of the electric field in the poled glass is an important factor determining the NLO properties produced during the poling. The study of this will lead to a better explanation and an understanding of the poling pro- cess. Up to now, the various methods used for probing the electric field distribution are optical for most of them. They measure, in fact, the $\chi^{(2)}$ distribution [maker fringe method ${ }^{4}$ and noncollinear frequency doubling $\left.{ }^{5}\right]$. Some of them use electric measurements utilizing a controlled perturbation: laser induced pulse pressure, ${ }^{6}$ thermal step method. ${ }^{7}$ The resolution of all these methods is of a few microns and thus the electric field close to the surface near the electrode cannot be measured. On the other hand, electron beam is the most adapted technique in surface studies and has been already used to probe the insulating properties of alumina ${ }^{8,9}$ in the first micron beneath the surface by measuring the variation of the secondary electron emission yield (SEEY). In this paper, we present new results obtained by SEEY technique from poled silica waveguides.

\section{EXPERIMENTAL DETAILS}

\section{A. Principle of secondary electron emission measurements}

The secondary electron microscope (SEM) Predictscan LEO 440 has been equipped with the optibeam optical system which allows the beam to be focused at a fixed position (point $A$ in Fig. 1) after the second condenser lens for any energy and current intensity required. The electron beam blanking unit (EBBU) is composed of two plates centered at point $A$, in order to avoid the lateral displacement of the beam during the blanking operation. The EBBU is activated by a function generator, which allows the delivering of pulses varying from $10^{-3} \mathrm{~s}$ to $40 \mathrm{~s}$ with a dose adjustable from a few $10^{-2} \mathrm{pC} /$ pulse to ca. $10^{8} \mathrm{pC} /$ pulse. 


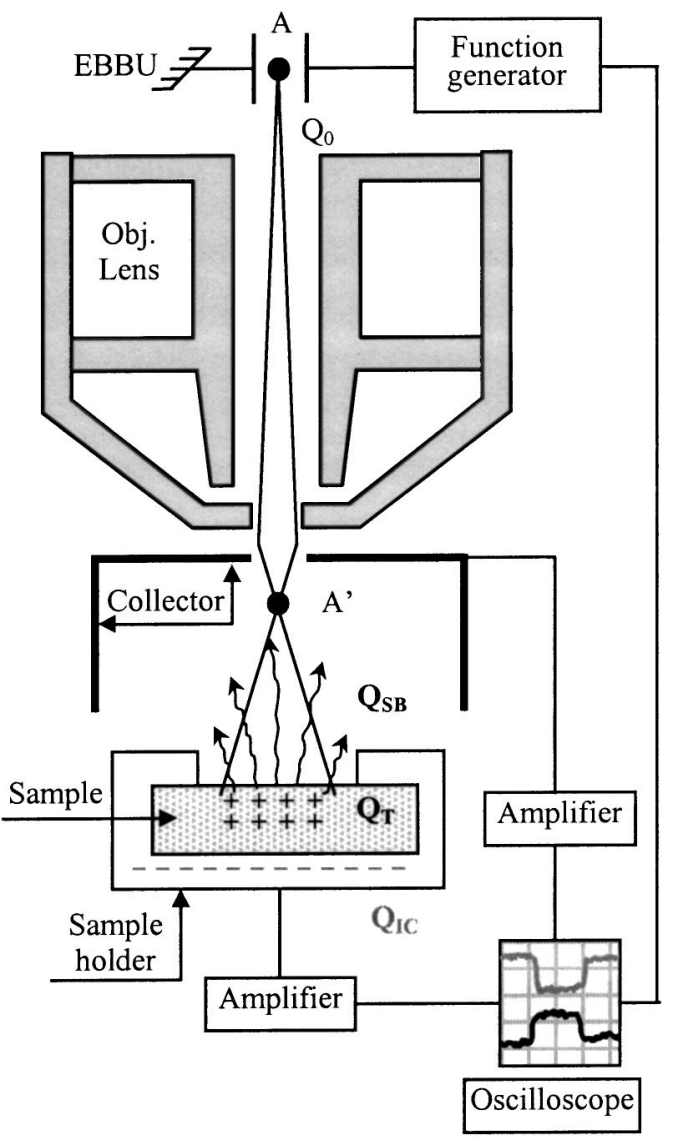

FIG. 1. Scheme of the bottom part of the microscope and principle of the induced current and secondary electron emission measurements. $Q_{\text {IC }}$ is the induced charge, $Q_{\mathrm{T}}$ the trapped charge, and $Q_{\mathrm{SB}}$ the secondary and backscattered electron charge.

Experiments are carried out in the spot mode with a defocused beam in order to obtain a uniform charging of the insulator.

The specimen chamber is equipped with two detectors, which collect the net charge $Q_{\mathrm{T}}$ created in the sample and the charge $Q_{\mathrm{SB}}$ of secondary and backscattered electrons released from the surface into the vacuum, respectively. The net charge created in the sample is deduced from the measurement of the charge $Q_{\mathrm{IC}}=-Q_{\mathrm{T}}$ induced on the metal sample holder. 8 The SEEY $\delta(D, J)$ is measured as a function of the injected dose $D(\geqslant 0.01 \mathrm{pC})$ or charge density $\sigma$ at a constant current density $J\left(\gtrsim 10^{+3} \mathrm{pA} \mathrm{cm}^{-2}\right)$. It is defined as

$$
\delta(D, J)=\frac{Q_{\mathrm{SB}}}{Q_{0}}=1-\frac{Q_{\mathrm{IC}}}{Q_{0}},
$$

where $Q_{0}=Q_{\mathrm{SB}}+Q_{\mathrm{IC}}$ is the injected charge. The intrinsic SEEY $\delta_{0}$, which is the yield of the uncharged insulator, is measured by injecting a very low dose $(<0.1 \mathrm{pC})$ on a large virgin area $\left(2.5 \times 10^{-3} \mathrm{~cm}^{2}\right)$, i.e., the deposited charge density is $<40 \mathrm{pC} / \mathrm{cm}^{2}$. When $\delta_{0}>1$ as it is in the case when the electron beam energy is $1100 \mathrm{eV}$, the sample charges positively and $Q_{\mathrm{IC}}<0$. When $\delta_{0}<1$ for an electron beam energy of $5 \mathrm{keV}$, the sample charges negatively and $Q_{\mathrm{IC}}>0$.

Then, charges accumulate in the insulator, producing an electric field beneath the surface, which induces a variation

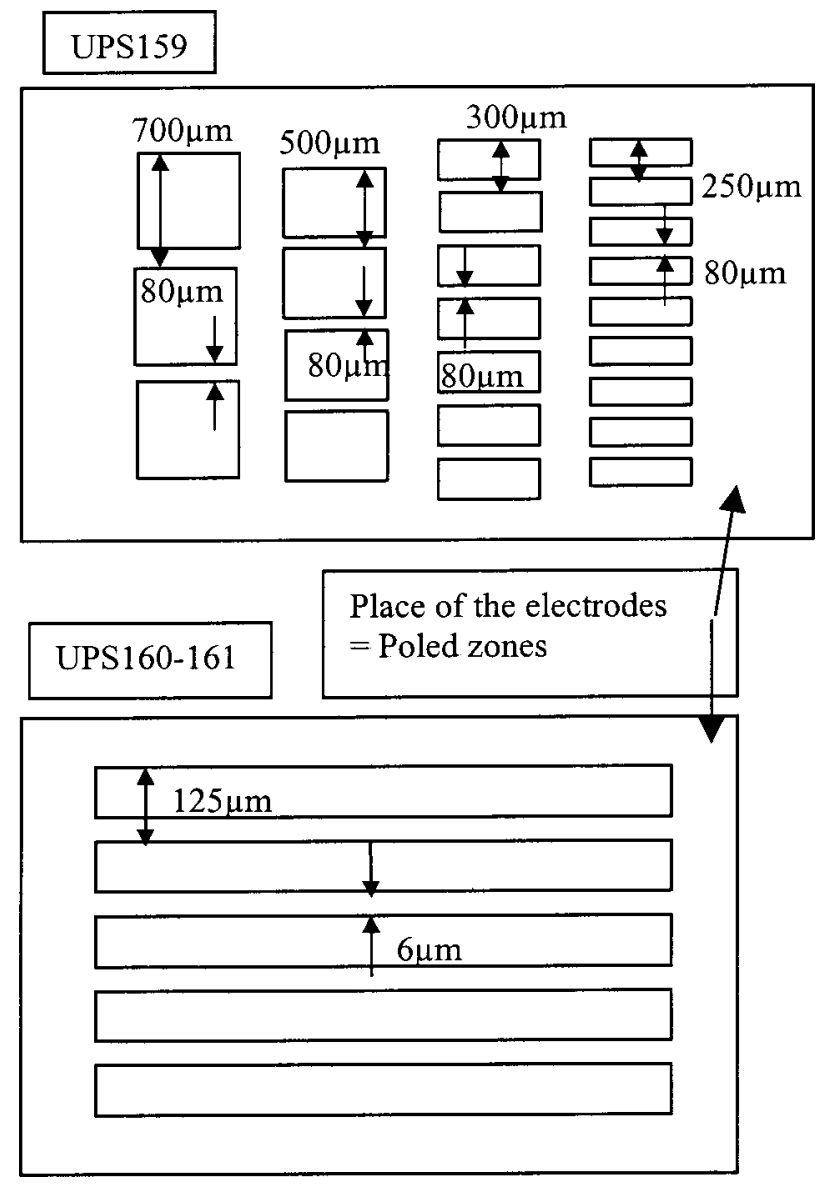

FIG. 2. Geometry of poling electrodes for UPS159 and UPS160-161.

of $\delta$ towards unity. This variation is characteristic of the charging properties of the insulator. This is why the SEEY investigation is a valuable method of characterization of insulators.

\section{B. Sample preparation}

Glass samples (UPS159, - 160, - 161) were prepared by plasma enhanced chemical vapor deposition and were investigated in the secondary electron emission mode of a SEM. The thin glass layer $(1.1 \mu \mathrm{m})$ deposited on a Si wafer substrate is Ge doped silica.

Comb shaped aluminum electrodes $(\sim 1.2 \mu \mathrm{m}$ thick $)$ were deposited on glass layer through a photoresist mask for the poling process. The geometry of the Al electrode is depicted in Fig. 2.

During a thermal poling process, a negative voltage ranging from -30 to $-100 \mathrm{~V}$ was applied across the samples when keeping the Si substrate grounded. Samples were poled for $15 \mathrm{~min}$ at $375^{\circ} \mathrm{C}$ and then the heating was stopped. The poling voltage was kept till the sample was cooled down to the room temperature.

In order to study the poled samples in the SEM, the $\mathrm{Al}$ electrodes were removed by a concentrated $\mathrm{H}_{3} \mathrm{PO}_{4}$ acid for $20 \mathrm{~min}$ at $75^{\circ} \mathrm{C}$. After that, reflection microscopy in white light and chemical analysis were performed to characterize 

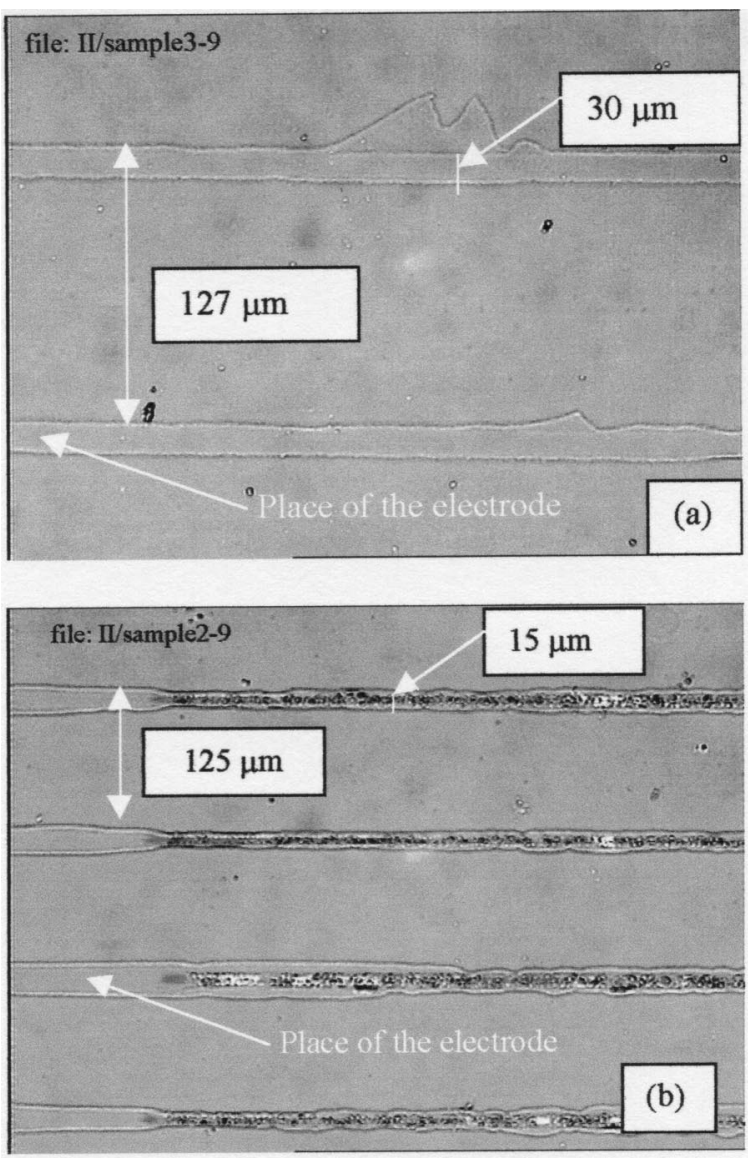

FIG. 3. Optical reflection microscopy with natural light. Magnification $=10 \times$ for (a) and $5 \times$ for (b). Photos show the effect of electrodes spreading on sample UPS 161 photo (a) and defects induced by poling process on sample UPS 160 photo (b).

the samples before SEEY measurements. The SEEY was measured on the samples not covered with a conductive layer.

\section{Reflection microscopy}

A precise location of the removed electrodes can be achieved by means of reflection microscopy due to the variations of the reflection coefficient between the poled and unpoled areas. As shown in Fig. 3, some irregularities at the poled-unpoled borderline [Fig. 3(a)], as well as damage [iridescent zones in Fig. 3(b)], can be easily seen. In order to separate the contribution from the chemical removal of the electrodes and from the poling process, we observed a sample that has not been poled (UPS 202) just after removing the electrodes. From the surface of this sample, it is very difficult to distinguish any variation of the reflection coefficient. This indicates that the chemical process with concentrated $\mathrm{H}_{3} \mathrm{PO}_{4}$ is a "soft" process that does not damage the surface, and the contrast observed in Fig. 3 is most likely due to the poling process. To reinforce this conclusion, measurements of the surface topography were performed and have been presented elsewhere. ${ }^{10}$ The relevant result for the present study is that a surface level increase appears when samples are poled but no change of level is detected after electrode removing when sample has not been poled.
Electron microprobe analysis on UPS 160 - series 3
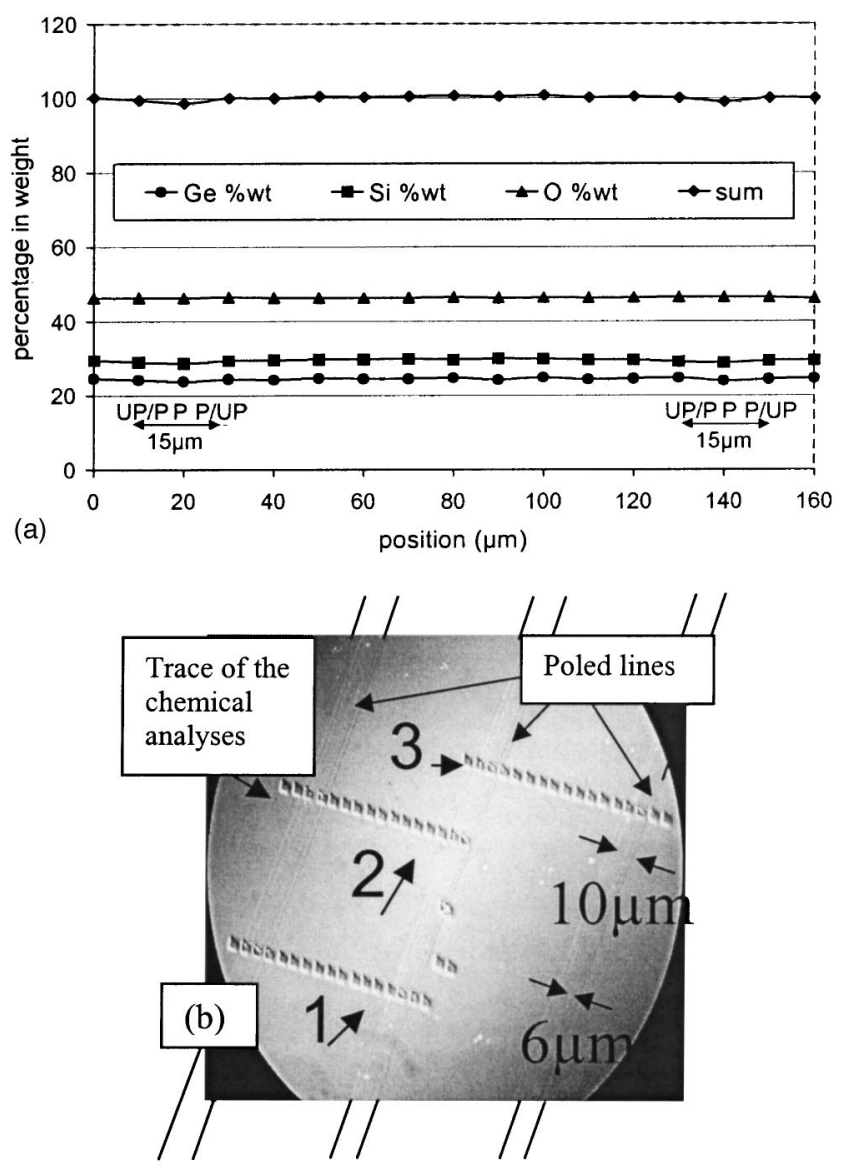

FIG. 4. Braga: (a) Photograph of UPS 160 taken in white light by reflection showing three poled lines and three chemical profiles including the one called series 3 . The quotation NP means unpoled region whereas the quotation $P$ means poled region. UP/P means that the measurement was done at the borderline. (b) Corresponding chemical analysis by EPMA through two poled lines of the series 3 . The places of the poled lines that are less contrasted than the traces of the chemical analyses are marked with black lines out of the photograph.

\section{Chemical composition characterization}

The chemical analysis was made by electron probe for microanalysis in a wavelength dispersive $\mathrm{x}$-ray configuration at a voltage of $10 \mathrm{kV}$ with an electron current of $50 \mathrm{nA}$. The luminescence x-ray lines were: $K \alpha(\mathrm{Si})=1740 \mathrm{eV}, K \alpha(\mathrm{O})$ $=530 \mathrm{eV}, \quad K \alpha(\mathrm{N})=409.9 \mathrm{eV}, \quad L \alpha(\mathrm{Ge})=1188 \mathrm{eV}, \quad$ and $K \alpha(\mathrm{P})=2015 \mathrm{eV}$. Figure 4 shows the profile of the chemical composition in the sample UPS 160 coated with carbon. The curves show the profile of $\mathrm{Ge}, \mathrm{Si}$, and $\mathrm{O}$ content. The average composition through poled and unpoled areas is the following: $\quad \mathrm{Ge}=24.33 \mathrm{wt} \%, \quad \mathrm{Si}=29.48 \mathrm{wt} \%, \quad$ and $\mathrm{O}$ $=46.23 \mathrm{wt} \%\left(\mathrm{Ge}_{0.24} \mathrm{Si}_{0.76} \mathrm{O}_{2+0.09}\right)$. Their sum in mass emphasizes a slight decrease in weight percentage of the Ge and Si components alongside the poled areas shown in the photograph [Fig. 4(b) and Fig. 6]. Because the sum is depressed at the place of the electrodes, other elements were sought for. Though, no Al diffusion from the electrode to the glass has been detected but a weak content of phosphorus inserted at the place of the electrodes and arising from the electrode removing treatment (see Fig. 5) was detected. 


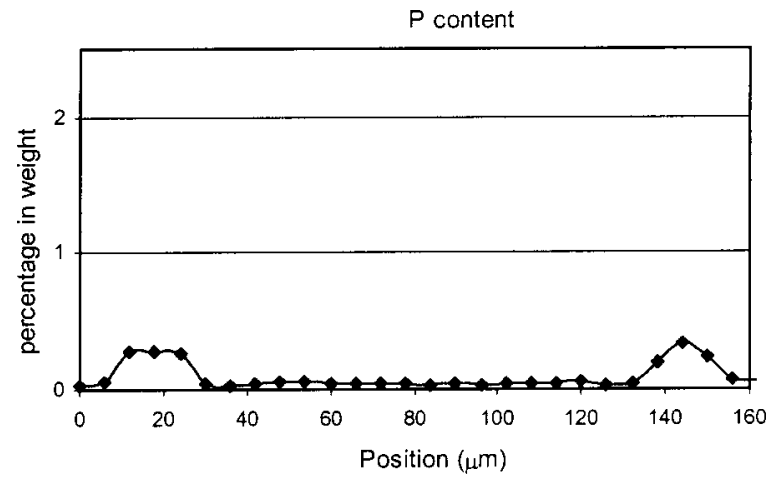

FIG. 5. EPMA chemical analysis of UPS 160: detail of the curve "Ge" and "SI."

However, the decrease of $\mathrm{Si}$ and $\mathrm{Ge}$ counts in the poled area in Fig. 6 is not compensated by an increase of oxygen or phosphorus content so that the "sum" value is not exactly $100 \mathrm{wt} \%$ in Fig. 7. This prevents a very quantitative estimate of chemical change induced by poling eventually. We can only say that chemical change is smaller than $1 \mathrm{wt} \%$. Nevertheless, these measurements point out that the effective volume analyzed is not exactly the same in the poled and unpoled areas or in the references.

Lastly, it appears that the change in composition is too small to give rise to the contrast observed in the photograph of the Fig. 4. However, the poling process can change the glass structure, changing the optical properties of the glass and thus the reflection coefficient. This point will be described in another paper.
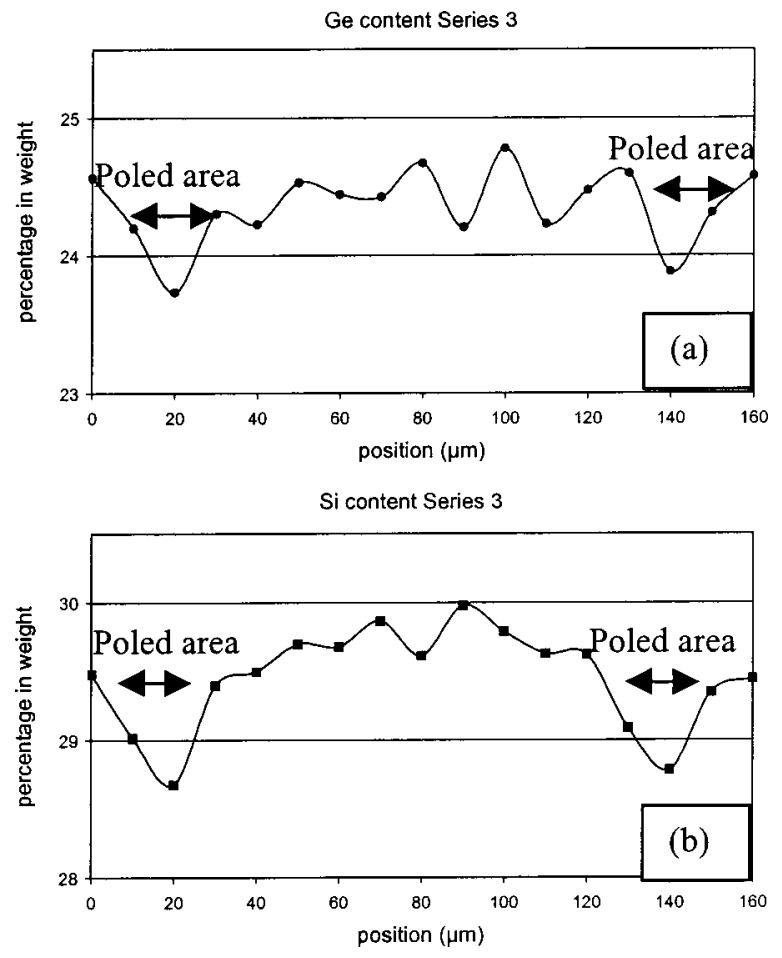

FIG. 6. EMPA chemical analysis of UPS 160: detail of the curve "O" and "sum."
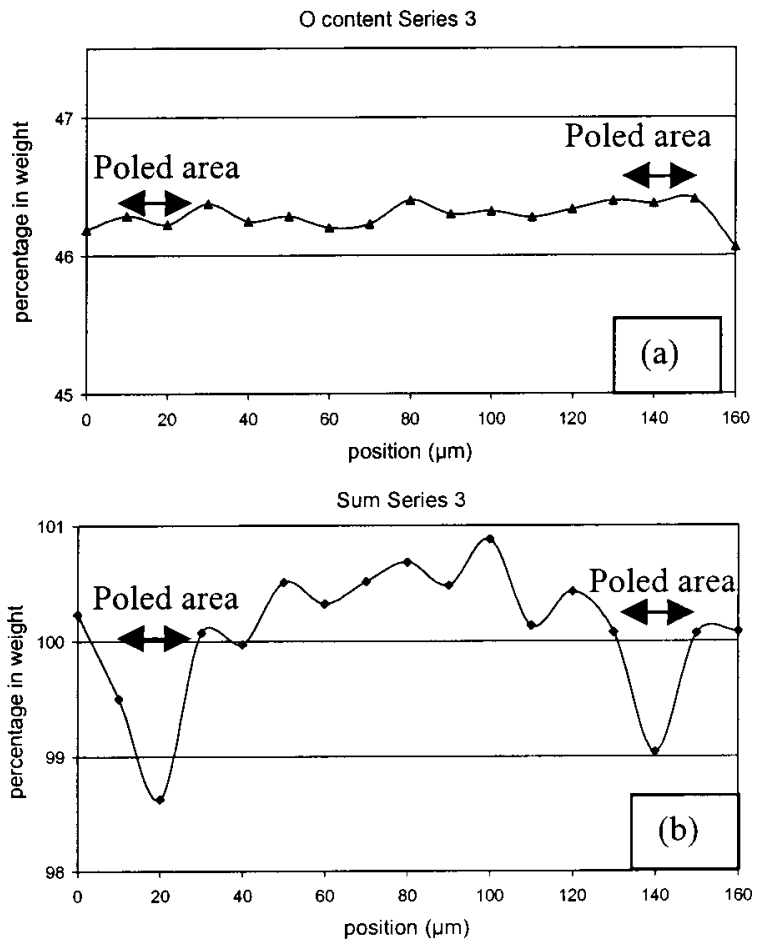

FIG. 7. Phosphorous content on UPS 160 through the poled lines. Compared Figs. 6 and 7 this measurement has been performed afterwards. It corresponds to $0.3 \mathrm{~mol} \%$ of $\mathrm{P}_{2} \mathrm{O}_{5}$.

\section{RESULTS: SEM OBSERVATIONS AND SEEY MEASUREMENTS OF UPS 159 SAMPLE}

SEM observations and SEEY measurements were performed for electron acceleration energy of $1100 \mathrm{eV}$ and 5000 $\mathrm{eV}$ in order to probe different depths, ${ }^{2} 22 \mathrm{~nm}$ and $530 \mathrm{~nm}$, respectively.

\section{A. Investigation at $1100 \mathrm{eV}$}

Secondary electron images presented in Fig. 8 were obtained by scanning a zone containing poled and unpoled regions of sample UPS 159. The energy and current of electrons impinging the sample are, respectively, $1100 \mathrm{eV}$ and 6 pA. At this energy, the intrinsic SEEY is larger than one (Fig. 9) so that the sample charges positively. In these conditions, the minimum possible injected charge density in a single scan is about $700 \mathrm{pC} \mathrm{cm}^{-2}$. After injection of a charge density of $38000 \mathrm{pC} \mathrm{cm}^{-2}$, a strong contrast appeared [Fig. 8(a)]: the poled regions are brighter than the unpoled ones which means the SEEY is larger in the poled regions than in the unpoled ones. On further scanning, the contrast increases first and then decreases until it almost vanishes.

This evolution is exemplified by performing an enlargement of the scanning area of Fig. 8(a) in order to reveal a new region, virgin of charges. After a single scan $(\approx 700$ $\mathrm{pC} \mathrm{cm}{ }^{-2}$ ) a very little contrast appears in the outer part of the image [Fig. 8(b)]. After the injection of $9000 \mathrm{pC} \mathrm{cm}^{-2}$ [Fig. $8(\mathrm{c})]$, the contrast becomes stronger in this region and goes on to increase as the charge density is increasing [Fig. 8(d)]. In the same time, the contrast in the inner scanned area decreases [Fig. 8(e)]. Finally, the brightness in the poled and unpoled areas tends to be the same [Fig. 8(f)] when the scanning charge density becomes very large. What it is seen, 


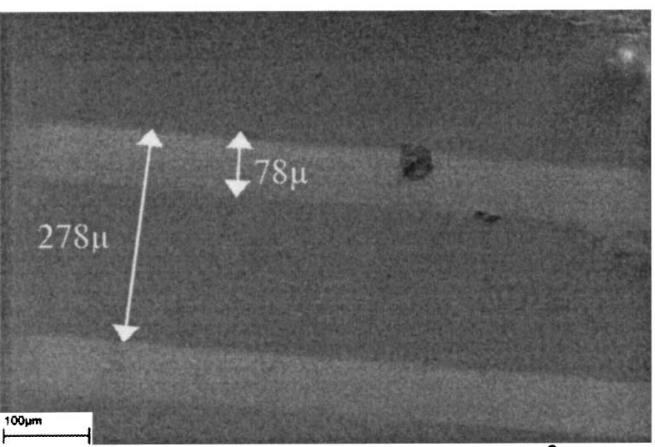

(a) after a scan of 38000 pC.cm ${ }^{-2}$

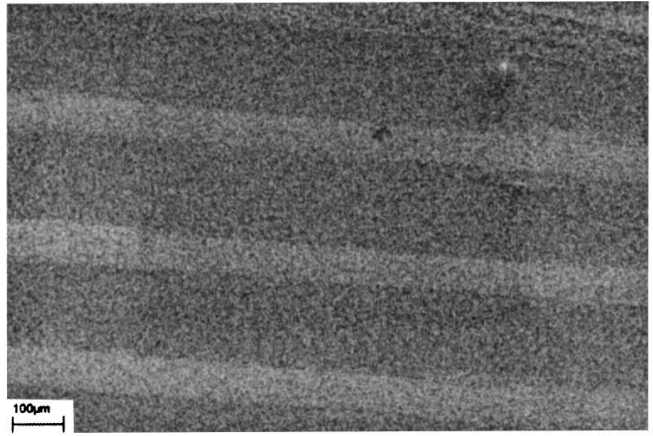

(c) Total charge density $=9000 \mathrm{pC} \cdot \mathrm{cm}^{-2}$ on the outer part.

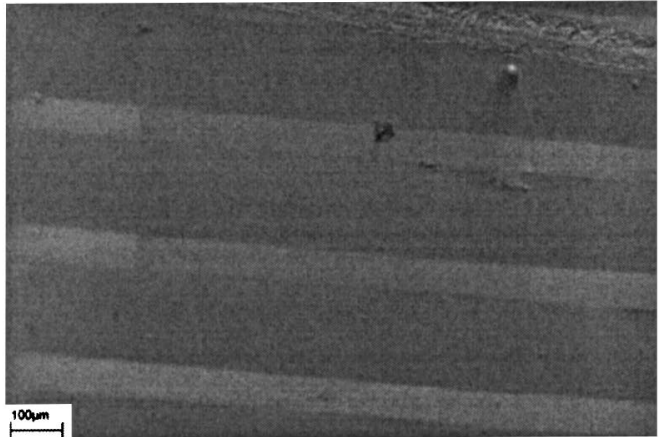

(e) Total charge density $=80000$ pC.cm ${ }^{-2}$ on the outer part.

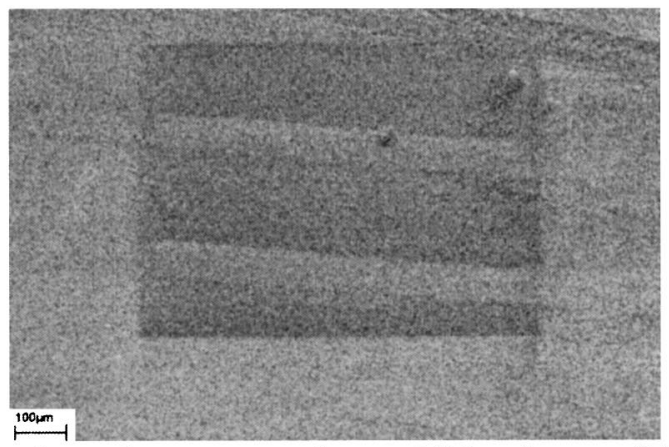

(b) after enlargement and one additional scan of 700 $\mathrm{pC} . \mathrm{cm}^{-2}$

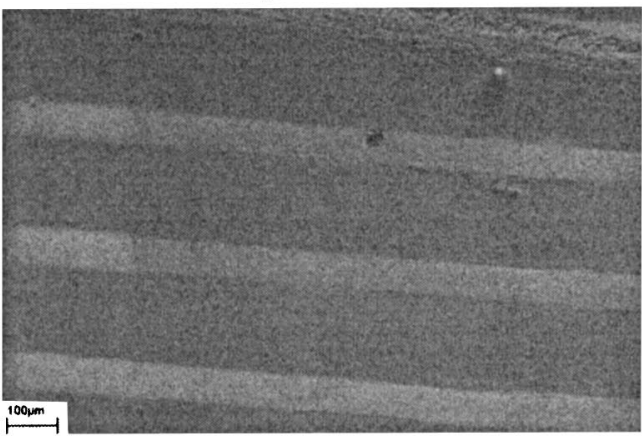

(d) Total charge density $=43600 \mathrm{pC} . \mathrm{cm}^{-2}$ on the outer part.

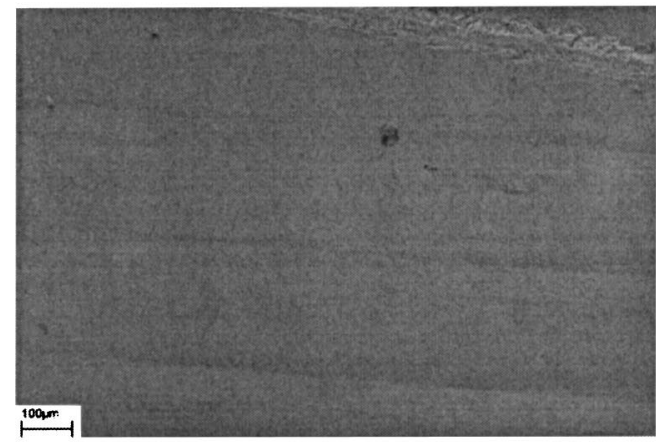

(f) Total charge density $=217000 \mathrm{pC} . \mathrm{cm}^{-2}$ on the outer part.

FIG. 8. Scans showing the variation of the contrast during irradiation. The poled strips are bright in Figs. 8 (a) to $8(\mathrm{e})$. $E=1100 \mathrm{eV}$; the injected charge densities are indicated for each image. The scale at the bottom left side of each photograph is $100 \mu \mathrm{m}$. (a) After a scan of $38000 \mathrm{pC} \mathrm{cm}^{-2}$; (b) after enlargement and one additional scan of $700 \mathrm{pC} \mathrm{cm}^{-2}$; (c) Total charge density $=9000 \mathrm{pC} \mathrm{cm}^{-2}$ on the outer part; (d) Total charge density $=43600 \mathrm{pC} \mathrm{cm}^{-2}$ on the outer part; (e) Total charge density $=80000 \mathrm{pC} \mathrm{cm}^{-2}$ on the outer part; (f) Total charge density $=217000 \mathrm{pC} \mathrm{cm}^{-2}$ on the outer part.

here, is the change of SEEY with the electron irradiation.

So, this behavior was quantitatively studied by measuring the evolution of the SEEY versus the charge density $\sigma$ on poled and unpoled regions separately (Figs. 9 and 10). Several experiments were carried out on poled and unpoled areas to determine, first, the average value of $\delta_{0}$ (value at the beginning of irradiation) and second, the variation of $\delta$ with respect to the implanted dose. Results show a very small difference of $\delta_{0 \text { av }}\left(\delta_{0}\right.$ average value) in the two areas: theses values are $\delta_{0 \text { av }} \quad($ poled region $)=1.75 \pm 0.13$ and $\delta_{0 \text { av }}$ (unpoled region) $=1.85 \pm 0.04$. This is consistent with the weak contrast observed in the external part of Fig. 8(b) corresponding to $700 \mathrm{pC} \mathrm{cm}^{-2}$. However, during electron bombardment, $\delta$ in the unpoled region decreases much faster than in the poled region (Fig. 9). In the unpoled region, the selfregulated regime characterized ${ }^{8,9}$ by $\delta=1$ is reached for $\sigma$ $\approx 5 \times 10^{+4} \mathrm{pC} \mathrm{cm}^{-2}$ (Fig. 9) whereas in the poled region, $\delta$ reaches unity after the injection of $\sigma \approx 8 \times 10^{+6} \mathrm{pC} \mathrm{cm}^{-2}$ (Fig. 10). Poled areas exhibit longer decay kinetics than unpoled ones. The kinetics observed here corresponds to the evolution of the contrast observed in the images presented in Fig. 8. In the last image [Fig. 8(f)], the charge densities injected in the outer and inner part are about the same: 2.17 $\times 10^{+5} \mathrm{pC} \mathrm{cm}^{-2}$ and $2.55 \times 10^{+5} \mathrm{pC} \mathrm{cm}^{-2}$, respectively. The contrast is thus the same in these two regions. This contrast is due to the difference in the SEEY between poled and unpoled areas represented in Fig. 9 for a charge density of $2.55 \times 10^{+5} \mathrm{pC} \mathrm{cm}^{-2}$. To obtain a complete vanishing of 

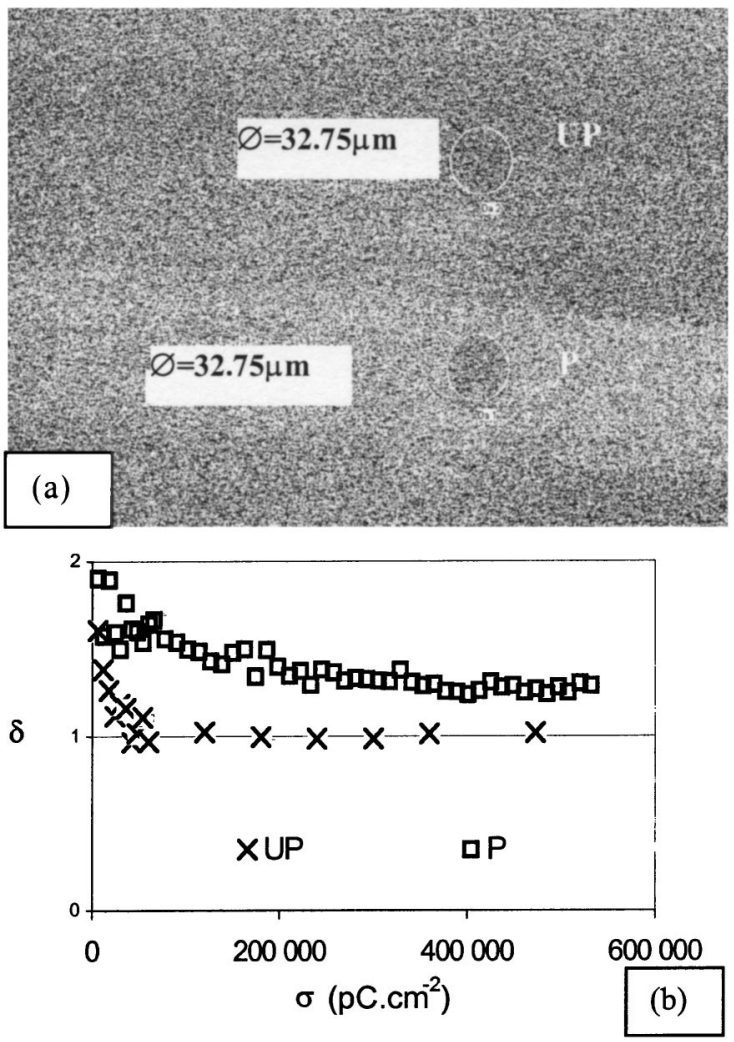

FIG. 9. Variation of the SEE yield $\delta$ of UPS 159 in poled and unpoled areas with the injected charge density $\sigma . E=1100 \mathrm{eV}, J=8.5 \cdot 10^{+5} \mathrm{pA} \mathrm{cm}^{-2}$ (a) the photograph showing the place of measurement, (b) the graph.

the contrast, that is, $\delta=1$ in both areas, a charge density of about $8 \times 10^{6} \mathrm{pC} \mathrm{cm}^{-2}$ is required. This implies about 11000 scans under the experimental conditions.

\section{B. Investigation at $5000 \mathrm{eV}$}

The same experiments as those reported in the section above were carried out on UPS 159 at the energy $E$ $=5000 \mathrm{eV}$. At this energy, $\delta_{0}<1$ so that the sample charges negatively (Figs. 11 and 12). The current of the electron beam impinging the sample was $12 \mathrm{pA}$. Figs. 11 and 12 represent the variation of $\delta$ with the injected charge density $\sigma$ in the poled and unpoled areas. From Fig. 12, it is clear for these electron acceleration energy that $\delta_{0}$ values are different in the two regions. In the unpoled region, the average value

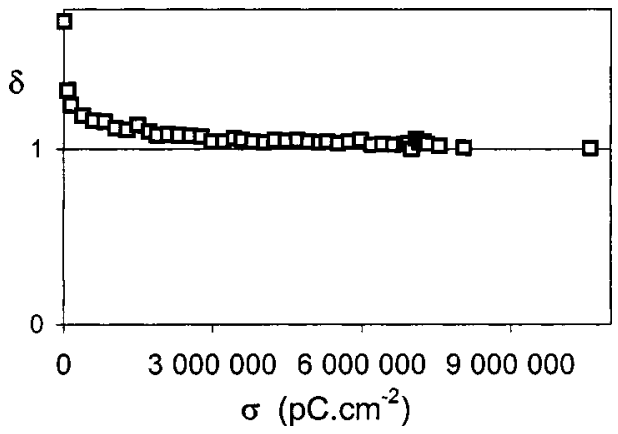

FIG. 10. Variation of the SEE yield $\delta$ of UPS 159 on a poled area at large injected charge density $\sigma . E=1100 \mathrm{eV}, J=8.5 \cdot 10^{+5} \mathrm{pA} \mathrm{cm}^{-2}$.

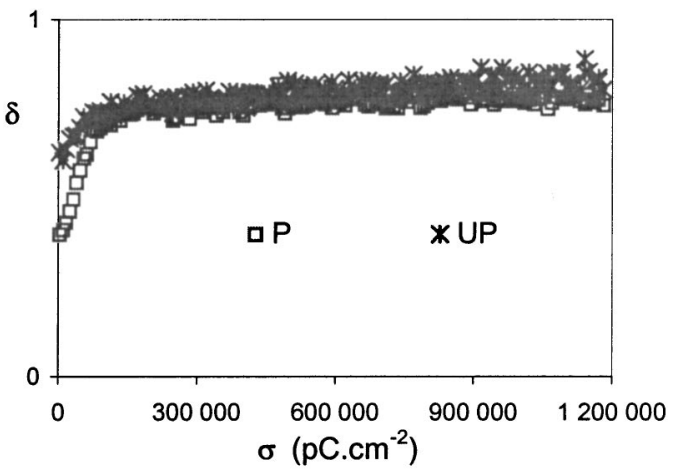

FIG. 11. Variation of the SEE yield $\delta$ of UPS 159 on unpoled (UP) and poled $(P)$ areas with the injected charge density $\sigma$ before the scans presented in Fig. 12(b). $E=5000 \mathrm{eV}, J=1.45 \cdot 10^{+6} \mathrm{pA} \mathrm{cm}^{-2}$.

of $\delta_{0}$ is $0.63 \pm 0.01$, the dispersion being due to the measurement precision. In the poled region, a dispersion of $\delta_{0}$ between 0.40 and 0.54 far beyond the instrument precision is observed. Figure 11 shows also a difference in the charging kinetics, which is faster in the unpoled region, like we have observed for electron energy of $1100 \mathrm{eV}$. At a high injected charge density (Fig. 12), the SEEY reaches a very stable steady value $\delta_{\infty}=0.84$ in the poled area for $\sigma>5$ $\times 10^{7} \mathrm{pC} / \mathrm{cm}^{2}$, whereas in the unpoled area, $\delta_{\infty}$ exhibits a rather large fluctuation around the value 0.95 for $\sigma$ $>10^{7} \mathrm{pC} / \mathrm{cm}^{2}$.

Two spots, on the unpoled and poled areas [spots 1 and 2 in Fig. 13(a)], respectively, received the same charge density $\sigma=1.2 \times 10^{6} \mathrm{pC} / \mathrm{cm}^{2}$. Then, the image presented in Fig. 13(a) was taken with a charge density of $2.3 \times 10^{5} \mathrm{pC} / \mathrm{cm}^{2}$ just after the irradiation of the two spots for obtaining the measurement shown in Fig. 11. The poled strip is darker than the unpoled one consistently with the lower $\delta$ coefficient appearing in Fig. 12 (the contrast is weak). It is noticed that the two spots are clearly visible (brighter) in Fig. 13(a) because they have received a higher charge density than the background and consequently their $\delta$ coefficient is larger (Fig. 11).

Figure 13(b) was taken with a charge density of 1.9 $\times 10^{6} \mathrm{pC} / \mathrm{cm}^{2}$, so spots 1 and 2 received a total charge den-

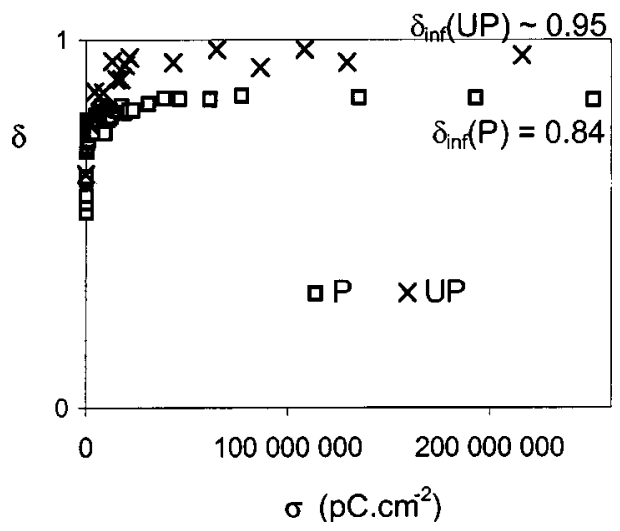

FIG. 12. Variation of the SEE yield $\delta$ of UPS 159 on poled $(P)$ and unpoled (UP) area for a large injected charge density $\sigma . E=5000 \mathrm{eV}, J$ $=1.45 \cdot 10^{+6} \mathrm{pA} \mathrm{cm}^{-2}$. 

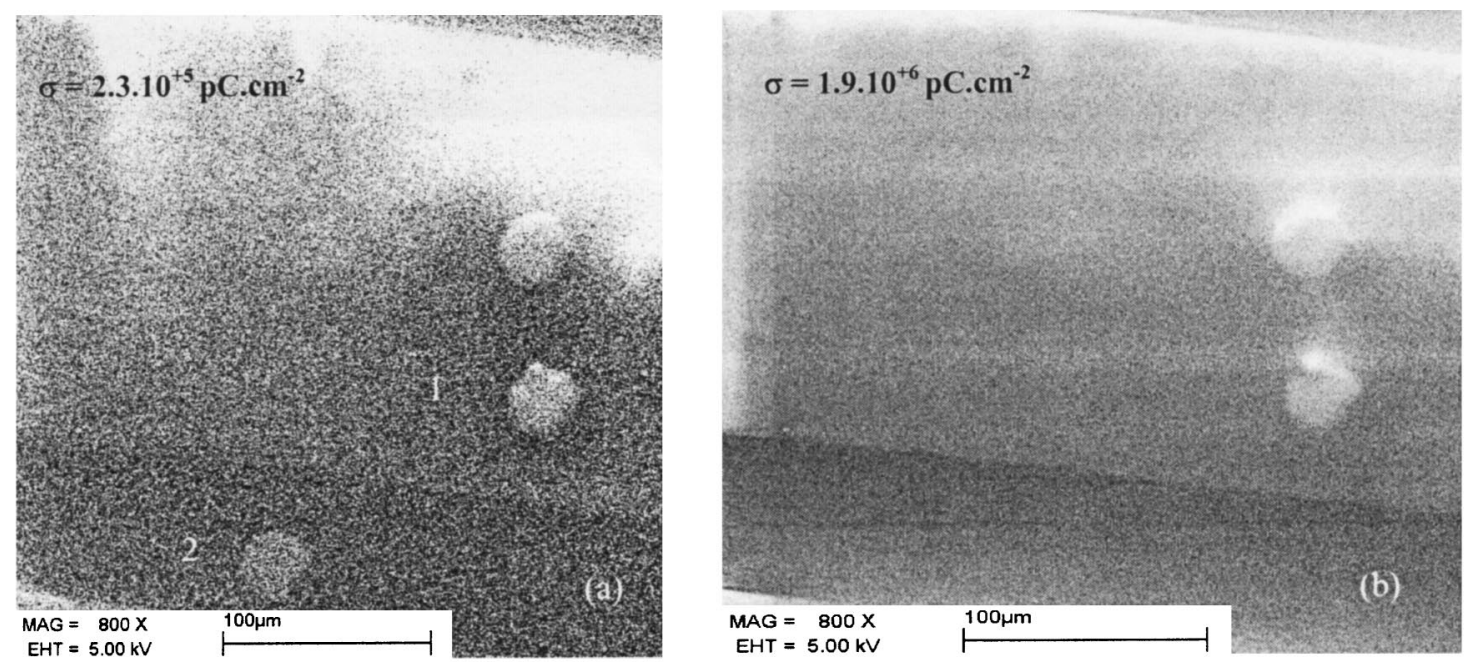

FIG. 13. Secondary Electron Emission images of UPS 159 obtained for two different injected charge densities (a) $\sigma=2.3 \times 10^{5} \mathrm{pC} / \mathrm{cm}^{2}$ and (b) $\sigma=1.9$ $\times 10^{6} \mathrm{pC} / \mathrm{cm}^{2}$ showing the variation of the contrast after the irradiation presented in Fig. 10. Electron beam energy $E=5000 \mathrm{eV}$. Spots 1 and 2 have been irradiated with $1.2 \times 10^{+6} \mathrm{pC} \mathrm{cm}^{-2}$ before being displayed by scanning.

sity of $(1.2+1.9) \times 10^{6} \mathrm{pC} / \mathrm{cm}^{2}$. We note that the contrast between poled and unpoled regions is better. This is consistent with the difference in the $\delta$ coefficient (the two $\delta$ curves separate). Now, it is noticed that the spot 1 on the unpoled area is brighter than in Fig. 13(a), whereas the one on the poled area has completely vanished in the background. Consistently with Figs. 11 and 12, in the unpoled region, the SEEY goes on to increase with the injected charge whereas for poled region it has leveled off. This clearly demonstrates that the poled and unpoled regions do not exhibit the same charging properties.

\section{DISCUSSION}

To understand the evolution of the contrast in Figs. 8 and 13 , we will examine the various contributions to the contrast in SEM, i.e., topography, chemistry, poling electric field, and glass structure modification due to poling.

\section{A. Topographic origin of the contrast}

It is well known that the SEEY is sensitive to the topography. In the present case, the topography of the sample surface is slightly changed under poling and this is described in details in another paper. ${ }^{10}$ Let us just mention here that the level of the surface in the poled region has moved up a few $\mathrm{nm}$ with respect to the unpoled one. If a contrast was due to that change in topography, it would have been observed just after one scan of $700 \mathrm{pC} / \mathrm{cm}^{2}$ for any electron acceleration energy, in particular, for electrons with energy $1100 \mathrm{eV}$, i.e., in the outer part of Fig. 8(b). In fact, there is no evidence of such a topographic contrast in this figure. Therefore any contrast between white and dark strips afterwards cannot be attributed to the topography. However, the dark lines that underline the border between poled and unpoled regions in Fig. 8(f) could be attributed to a shadow effect. This line is not observed in Fig. 13(b) where the electron beam energy and the electron penetration depth $R(E=5000 \mathrm{eV}, R \sim 530 \mathrm{~nm})$ are larger than in Fig. 8(f) $(E=1100 \mathrm{eV}, R \sim 20 \mathrm{~nm})$.

\section{B. Chemical origin of the contrast}

A contribution from the chemical composition to the contrast also has to be excluded for a similar reason as previously mentioned, i.e., the contrast will exist from the beginning for any electron acceleration energy, in particular, for electrons with energy $1100 \mathrm{eV}$, i.e., in the outer part of Fig. 8(b). Especially, the small $P$ pollution or an effect of Al doping from the electrode would have been detected at the beginning if they exhibited an effect on SEEY. We can thus conclude that there is no effect of chemical composition difference if there is any.

\section{Poling electric field induced contrast}

The objective of thermal poling as described in the Introduction is to store a strong electric field in building a space charge by separating negative and positive charges. Thus, there could be an electric field in the poled region and not in the unpoled one before electron irradiation. As the SEEY is sensitive to the internal electric field, a contrast is expected due to the difference of the intrinsic $\delta_{0}$ values between the two regions. If the poling field is directed outward [such as in Fig. 14(b)], it prevents secondary electrons to escape and consequently $\delta_{0}$ in the poled region is less than in the absence of the poling field at both energies 1100 and $5000 \mathrm{eV}$. If the poling field is directed inward, the extraction of electrons is favored and consequently $\delta_{0}$ in the poled region is larger than in the unpoled region [Fig. 14(a)].

Despite the rather large dispersion of the SEEY values in the poled regions, it is noticed that, in average, $\delta_{0}$ in the poled regions is less than in the unpoled ones, at both energies: 1.75 against 1.85 at $1100 \mathrm{eV}$ and 0.47 against 0.63 at $5000 \mathrm{eV}$. However, thanks to the different electron penetration depths, we deduce that the difference is smaller at the surface than half a micron below. The decrease of $\delta$ arises from an attracting field for the secondary electrons and it is thus concluded that poling has left an internal electric field directed outwards on a depth of the order of the electron 


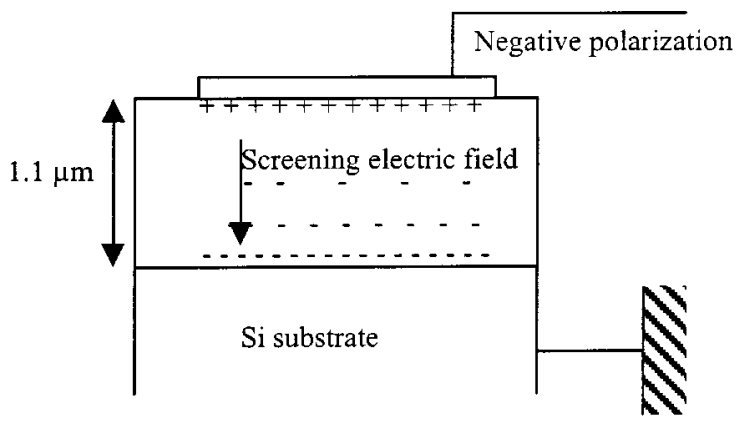

(a)

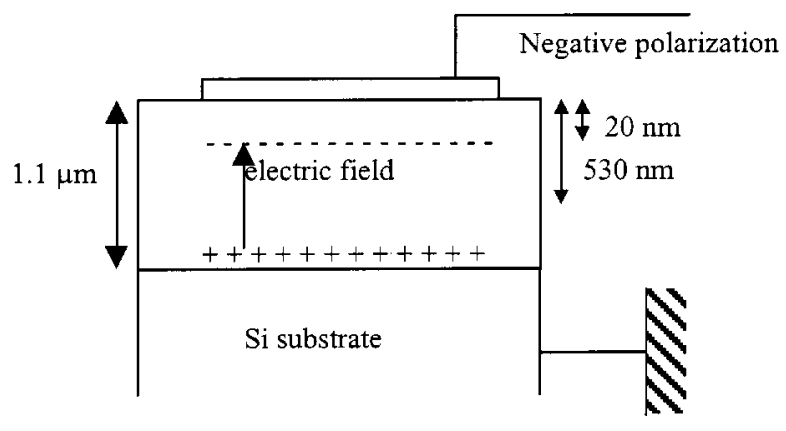

(b)

FIG. 14. Electric field distribution (a) scheme for the expected electric field and the space charge distribution deduced from bulk thermal poling experiment. (b) Actual distribution due to poling taking into account the SEEY results for an electron acceleration of $1100 \mathrm{eV}$ and for $5000 \mathrm{eV}$.

implantation depth $(530 \mathrm{~nm}$ for $5000 \mathrm{eV})$. Furthermore, the large fluctuations of $\delta_{0}$, that we have detected in the poled regions, indicate that the field is not uniform.

The issue now is about this electric field orientation. This is surprising if we note that the poling was negative in this sample (i.e., the surface electrode was negative and the substrate positive). Let us recall that during the poling process $\left(30-100 \mathrm{~V} / \mu \mathrm{m}, 300^{\circ} \mathrm{C}, 0.5 \mathrm{~h}\right)$, some species are mobile and move to the electrodes of polarity opposite to their sign. For instance, $\mathrm{Na}^{+}$migrates to the negative electrode and leaves behind negative ions trapped in the network, ${ }^{1}$ in such a way that a screening electric field built on mobile species ${ }^{11}$ would lead to the case depicted in Fig. 14(a) (case met in bulk thermal poling) with the electric field directed inwards.

The above results are thus not consistent with this description. Recently, it has been shown that charge injection is possible from the electrodes, ${ }^{12}$ like electrons from the negative electrode or electron pumping from the positive one that is equivalent to positive charge injection [see Fig. 14(b)]. Of course, in this condition, the applied (external) electric field is not screened but is stored with the same orientation. This process is just limited by electric resistivity of the insulator and the thermal electron mean free path. As a matter of fact, Quiquempois et al. ${ }^{11}$ have shown that efficient electric field screening is not possible in a thin layer as it required a large charge separation. Thus, we confirm this theoretical result. However, these authors showed that charge injection destroys the screening charge but in our case, the charge injection is the most efficient process of space charge formation leading to storage of external dc electric field. In the case of screening, the induced electric field overcomes the external one by several orders of magnitudes. Here, it is too small and in fact, another electric field is formed by charge injection during poling process. This one cannot overcome the external electric field. It is thus necessary to increase the external one for obtaining large internal one.

\section{Glass structure modification induced contrast}

We are now interested in the dynamic evolution of $\delta$ that is conditioned by charge mobility property in the insulator. Usually, if the sample is insulating like in the unpoled areas, the charge deposited during electron irradiation leads at each shot to a trapping of a fraction of this charge. This trapped charge accumulates, producing an electric field beneath the surface which induces a change of the SEE yield until a steady state is reached (the so-called self-regulated regime). ${ }^{8,9}$

Quantitatively, for $E=1100 \mathrm{eV}$, the self-regulated regime in unpoled areas $(\delta=1)$ is reached when the deposited charge is $\sigma=5 \times 10^{+4} \mathrm{pC} \mathrm{cm}^{-2}$ (see Fig. 9). This corresponds to the following net trapped charge $\sigma_{\mathrm{T}}=1.25$ $\times 10^{+4} \mathrm{pC} \mathrm{cm}^{-2}$ deduced from by the following integration:

$$
\sigma_{\mathrm{T}}=J_{\mathrm{T}} \int_{0}^{\infty}[\delta(t)-1] d t,
$$

where $J_{\mathrm{T}}$ is the primary current density. Using simple electrostatic law, we calculate the additional surface potential at the center of the spot assuming an equivalence with a uniformly charge disk by the following formula:

$$
V_{\mathrm{S}}=\frac{2 a}{\left(1+\varepsilon_{\mathrm{r}}\right) \varepsilon_{0}} \sigma_{\mathrm{T}},
$$

where $\varepsilon_{\mathrm{r}}$ and $\varepsilon_{0}$ are, respectively, the relative permittivity of the material $\left(\varepsilon_{\mathrm{r}} \sim 4\right.$ for $\left.\mathrm{SiO}_{2}\right)$ and the vacuum permittivity. With " $a$ " $\sim 16.3 \mu \mathrm{m}$, the radius of the irradiated area, we find $V_{\mathrm{S}} \sim 57.4 \mathrm{~V}$ in the unpoled area.

If we perform the same calculation in the poled area for $\sigma=8 \times 10^{6} \mathrm{pC} / \mathrm{cm}^{2}$ (Fig. 10), we find $V_{\mathrm{S}} \sim 2730 \mathrm{~V}$ which is beyond the incident electron beam energy. Of course, this is not possible at the sight of the images presented in Fig. 8 because such a surface potential would lead to a strong distortion of these images. The potential is therefore much lower, probably of the same magnitude as in the unpoled region. The other possibility leading to a stable $\delta \neq 1$ is the existence of a spreading of the charge in the bulk. It is thus concluded that charges do not stay confined within the spot area. This means that in the poled region, the conductivity allows the net positive charges to be eliminated from the spot area, which is not the case in the unpoled region where they are firmly trapped. We thus have to consider that poling process breaks the insulating properties of the glass. As a matter of fact, the surface potential can decrease a lot if the charge $\sigma_{\mathrm{T}}$ is decreased due to migration to the bulk (even if the sample is electrically isolated). It is even more efficient if the sample is electrically grounded. This conclusion is reinforced by the study at $5000 \mathrm{eV}$.

At $5000 \mathrm{eV}$, the SEEY reaches steady state values less than unity, equal to $\delta_{\infty}=0.95$ and $\delta_{\infty}=0.84$, respectively, in 
the unpoled and poled areas. This means that the sample is continuously charging with electrons in proportion to the injected dose. If all these electrons were trapped in the spot areas under the electron penetration depth $(1 / 2 \mu \mathrm{m})$, giant negative surface potentials would be obtained from Eqs. (2) and (3). It would be much higher than the acceleration potential of the electron beam. Clearly, here also, the charge spreads out of the irradiated area using charge relaxation processes in order to limit the surface potential. Looking at Fig. 13, the difference in the contrast evolution of the two spots 1 and 2 suggests that two different relaxation processes regulate the surface potential in unpoled and poled regions.

\section{E. Charge relaxation processes in unpoled silica}

It is observed in Fig. 12 that $\delta$ reaches a steady state at around $2 \times 10^{7} \mathrm{pC} / \mathrm{cm}^{2}$ and exhibits chaotic fluctuations. The large rate for $\delta$ to reach the steady state reveals that the net electron charge injected at $5000 \mathrm{eV}$ in unpoled silica is stable in time, which means that electrons are firmly trapped. However, as $\delta$ is smaller than 1 , charges accumulate. [This regime requires a large amount of charges trapped in depth to be reached because the penetration depth at $5 \mathrm{keV}$ is large face to the secondary electron escape depth]. But, the accumulation of charges also produces a strong electric field between the sample surface and the silicon substrate. If this field reaches the characteristic detrapping field of silica (about 5 $\mathrm{MeV} / \mathrm{cm}$ ), before the self-regulated regime is reached, a detrapping process takes place leading to a large expansion of the trapped charge distribution and a reduction of the surface potential. In other words, the surface potential in unpoled silica is regulated by a succession of trapping/detrapping events leading to a steady state value $\delta_{\infty}=0.95$ of the SEEY. The chaotic character of the process gives rise to large fluctuations of the surface potential and consequently to the $\delta_{\infty}$ value as observed in Fig. 12.

\section{F. Charge relaxation processes in poled silica}

It is observed in Fig. 12 that $\delta$ reaches a steady state at $5 \times 10^{7} \mathrm{pC} / \mathrm{cm}^{2}$ and then remains stable at a value departing from 1 clearly (0.84). This large departure shows that the net electron charge injected at $5000 \mathrm{kV}$ in poled silica is not stable in time, which means that electrons are mobile and leave the spot area. The test of stability will be described in details elsewhere. Here, the nonstability of the injected charge is attested by the fact that spot 2 vanishes as irradiation dose increases, contrary to spot 1 . Thus, the surface charge which regulates the surface potential results from a balance between the flux of incoming electrons and the flux of mobile electrons leaving the spot area. This competition which depends on the current density leads to an equilibrium with a steady state value $\delta=0.84$ which remains less than unity. In other words, in poled silica, the surface potential is regulated by the mobility of electrons. As this process is soft, compared to the abrupt detrapping events, the steady state value $\delta_{\infty}$ is weakly noisy. A calculation is underway to give an estimate of the surface potential as a function of $\delta_{\infty}$.

\section{G. Difference between poled and unpoled area in EPMA measurement}

The processes, involved in the EPMA measurements described in Sec IIC, are correlated with the processes described above. The $\mathrm{x}$ rays used in this method are emitted on the incident electron pathway like another relaxation process than secondary electron emission. The escape depth is somewhat larger ca. $1 \mu \mathrm{m}$. But the depth of origin depends on their energy, in fact. For oxygen dosage, we used $K \alpha$ line at $530 \mathrm{eV}$, whereas for $\mathrm{Si}$ or $\mathrm{Ge}$ ones, the lines were $K \alpha$ at 1740 and $L \alpha$ at $1188 \mathrm{eV}$ and thus photons from oxygen arise from a layer whose depth is different from the photons from Si $K \alpha$. As incident electron penetration depth varies with the electric field in the sample produced by the electron implantation, this induced electric field depends on the conductivity property of the glass at the place of measurement. As we have shown that conductivity is larger in the poled region, the electric field is smaller than in the unpoled regions. The repulsive electric field is smaller and thus the probed depth is therefore larger. This leads, therefore, to a smaller counting in the poled regions. This effect is not identical for different $\mathrm{x}$-ray energy and the resulting total wt \% departs from 100 . This effect is currently more deeply studied because it leads to variation of chemical content determination depending on the elaboration method for glasses of the same chemical composition.

\section{CONCLUSIONS}

We have measured SEEY in poled and unpoled regions of the same sample for detecting the electric field induced by thermal poling. We have detected an electric field in the upper half of the sample thickness and we have found a larger conductivity resulting from a perturbation of the glass structure by the poling process. We have detected an electric field in the same direction as the poling field. This confirms theoretical models and explains experimental features of the poling in layer configuration.

In thermal poling of bulk sample, the charges in the thermal poling process are recognized to be carried by $\mathrm{Na}$ and/or $\mathrm{H}$ traces. Those charges migrate towards negative electrode, negative charges being trapped in the glass network. ${ }^{5}$ The screening of the external electric field progresses with the charge separation. In silica, the positive charge was previously close to negative centers like negative nonbonding oxygen. Because positive charges are in a low concentration and not very mobile, it is difficult to reach large values ${ }^{11}$ of the distribution of the electric field induced (the screening field) in a $1.1 \mu \mathrm{m}$ sample.

At the same time, another process occurs: charge injection. The electric field used for the sample UPS 159 is -30 $\mathrm{V} / 1.1 \mu \mathrm{m}$. This large value allows the injection of electrons from the metal to the glass and pumps some other electrons from the glass to the silicon substrate. This process makes a charge separation on a small distance. SEEY is, therefore, a suitable method for detecting this electric field.

We point out also by SEEY measurements that the insulating layer poled is perturbed enough to lose a part of its insulating character. There is probably creation of defects 
under the strong electric field, i.e., $30 \mathrm{~V} / \mu \mathrm{m}$. The number of structural defects can be so large that it leads to higher electron conductivity. Such a large electric field magnitude is to be avoided because it appears to decrease the charge stability. Lastly, we point out unambigously the effect of the built in electric field on the chemical analysis of an insulator.

\section{ACKNOWLEDGMENT}

This work has been conducted in the framework of Research Training Network ODUPE (www.odupe.dk) from the European Commission, HPRN-CT-2000-0045 with a financial support from Contract GLAMOROUS (www.acreo.se/ glamorous) of the European Commission, (Grant nos. IST2000-28366), and from Ministère de la Recherche et des Nouvelles Technologies.

${ }^{1}$ R. A. Myers, N. Mukherjer, and S. R. J. Brueck, Opt. Lett. 16, 1732 (1991).

${ }^{2}$ W. Margulis, F. C. Garcia, E. N. Hering, L. C. Guedes Valentes, B. Le- sche, F. Laurell, and I. C. S. Carvalho, MRS Bull. Nov., 31 (1998).

${ }^{3}$ A. E. Grün, Z. Naturforsch. 12A, 89 (1957).

${ }^{4}$ J. Jerphagnon and S. J. Kurtz, J. Appl. Phys. 41, 1667 (1970).

${ }^{5}$ H. Guillet de Chatellus, S. Montant, and E. Freysz, Opt. Lett. 25, 1723 (2000)

${ }^{6}$ P. G. Kazansky, A. R. Smith, P. S. J. Russell, G. M. Yang, and G. M. Sessler, Appl. Phys. Lett. 68, 269 (1996).

${ }^{7}$ F. Odiot, I. Marc, O. Granier, A. Michez, and A. Toureille, Third French Italian Symposium on $\mathrm{SiO}_{2}$ and Advanced Dielectrics, Fuveau, France, June 2000 (Laboratoire de Physique de la matière de l'INSA, Lyon, 2000).

${ }^{8}$ G. Blaise and D. Braga, ISE 11 Conference, Melbourne, Australia, 2002.

${ }^{9}$ D. Braga and G. Blaise, Fourth International Conference on Electric charges in non-Conductive materials, Tours, France, 2001 (Societé Française du Vide, 2001), pp. 326-327.

${ }^{10}$ B. Poumellec, Y. Ren, and M. Kristensen, Bragg Gratings, Photosensitivity and Poling, Monterey, CA, USA, 2003 (OSA, Washington DC, 2003), pp. 165-167.

${ }^{11}$ Y. Quiquempois, G. Martinelli, P. Duthérage, P. Bernage, P. Niay, and M. Douay, Opt. Commun. 176, 479 (2000)

${ }^{12}$ A. Kudlinsky, G. Martinelli, Y. Quiquempois, and H. Zeghlache, Bragg Gratings, Photosensitivity and Poling, Monterey, CA, USA, 2003 (OSA, Washington DC, 2003), pp. 213-215. 